\title{
The Research of Character Analysis of Mechanical Filtering Material in High-Overload Environment
}

\author{
Yunbo Shi, Yuanying Qian, and Jie Zhu
}

\begin{abstract}
The material parameters of the filtering used the acceleration sensor was studied. In order to eliminate the damage that produced by high-frequency noise to acceleration in the process of weapon penetration, a filter and buffering was used to protect the sensor. Through theoretical analysis and finite element simulation, the relationship between the material parameters of the filter and the acceleration signal was described. The results show that the parameters of the filter material have a great influence on the filtering effect.
\end{abstract}

Index Terms-Simulation, penetration, mechanical filter, LS-DYNA.

\section{INTRODUCTION}

Micro mechanical accelerometer as a kind of inertial component was widely applied in the automatic control, vehicles, vibrating test, aerospace and other fields. In order to measure acceleration of different impact environment, high $\mathrm{g}$ value accelerometer is paid close attention to. Penetrating weapon usually penetrate hard target over hundreds of meters per second rate, and its acceleration can reach tens of $g$ values or the hundreds of thousands of $g$ value. So it requires that changed process of instantaneous acceleration can be quickly and correctly measure by sensor. At the same time, the sensor bear shock by high-overload. But in actual penetrating process, many high frequency noise signals from bullet is loaded into the rigid body penetrating acceleration signal, when bullet is squeezed by hole wall. So the acceleration signal produces shock. And the acceleration peak increase, result in instantaneous high $\mathrm{g}$ value can damage the accelerometer structure. In order to filter high frequency noise signals and analysis actual situation, a filtering mat is set up for filtering and protecting sensor.

This paper filters used for high $g$ value accelerometer was studied and the factors influenced the effect of filters were analyzed. The purpose is to find the matching materials which can be applied to different shaking environment.

\section{METHOD OF WORKING}

According to the different vibration source, vibrating

Manuscript received January 12, 2013; revised March 28, 2013.

Yunbo Shi is with the Key Laboratory of Instrumentation Science and Dynamic Measurement of Ministry of Education, North University of China, Taiyuan, 030051, China (e-mail: y.b.shi@126.com).

Yuanying Qian is with the Science and Technology on Electronic Test and Measurement Laboratory, North University of China, Taiyuan, 030051, China (e-mail: sufanqyy@163.com).

Jie Zhu is with the Science and Technology on Electronic Test and Measurement Laboratory, North University of China, Taiyuan, 030051, China (e-mail: zhujie@126.com). isolation include active vibrating isolation and passive vibrating isolation. Active vibrating isolation mean that vibration source is the machine itself; Passive vibration isolation mean that vibration source come from the motion of base. Passive vibrating isolation is that take measures to reduce vibration transferred to system from the outside world.

In the system vibration process, damping can consume vibration energy, rapidly weaken transient vibration, reduce the amplitude of forced vibration, avoid to producing the vibration of itself impelled and reduce energy of structure vibration transfer ability. Therefore, damping is the important means to control vibration. Increasing damping in the system, there are a variety of methods to achieve it, such as installing damper, using high internal resistance material for parts, setting up viscous materials, increasing the relative friction, etc. Considering the accelerometer itself volume is very small, the viscous damping materials are installed to reach effect of filtering vibration in this paper.

\section{THEORY OF VIBRATION}

Accelerometer and buffer, can regard as a two degree of freedom vibration system. Mathematical structure model is the following figure. We suppose that initial velocity and displacement of the system are zero. Assume that the initial displacement is zero. There is no compression or preloaded between $\mathrm{ml}$ and the base [1] and [2].

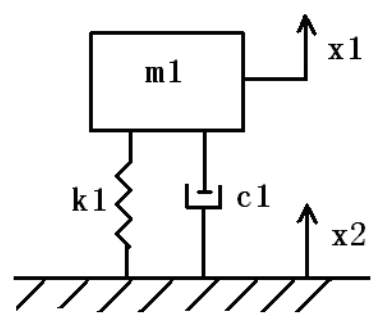

(a) two degree of freedom vibration

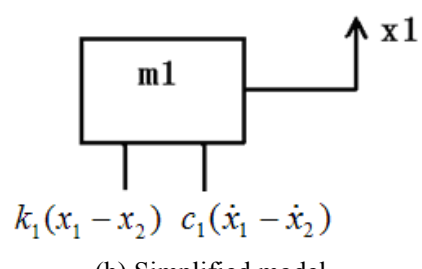

(b) Simplified model

Fig. 1. Shaking mathematical model.

Now, $m_{1}$ is called mass of accelerometer, $k_{1}$ is rigidity, $c_{1}$ is damping, $x_{1}$ is absolute displacement of $m_{1}, x_{2}$ is vibrating displacement. Assumed that the motion of base is 


$$
x_{2}=x \sin \theta t
$$

Relative displacement of $m_{1}$ is $x_{\Delta}=x_{1}-x_{2}$.

We claim base to fulfill $x_{\Delta}<x_{2}$, mean the vibration of the structure is less than the vibration of the basement. The equations motion of finishing system is:

$$
m \ddot{x}_{1}+c_{1}\left(\dot{x}_{1}-\dot{x}_{2}\right)+k_{1}\left(x_{1}-x_{2}\right)=0
$$

From the (2) we can see that in the movement behavior of $m_{1}$ is connected with $k_{1}$ and $c_{1}$. And the two parameters have close relationship with density and Young's modulus of material. In material damping constant, the greater the rigidity, the greater filter cutoff frequency [3]. Therefore, the material density and Young's modulus change, filtering effect also change.

\section{RESUlts AND Discussion/NumERICAL SimUlation}

In the projectile penetrating target process, signal acceleration sensor measured has a lot of noise, which contain a plenty of structure vibration signal produced by projectile when it is extruded, and a plenty of noise which is produced inside projectile. The high frequency noise not only enhance the peak of acceleration curve, but also damage structure of the sensor, in the other words, the sensor sensitive structure beam will fracture. In order to reduce the noise, a thickness of $0.5 \mathrm{~cm}$ filter is used at bottom of the sensor. And study how it influences the acceleration signal. Changing the parameters of filter, the influence of the filter different parameters is studied for the acceleration signal. We use the ogive nosed projectiles whose length-diameter ratio is $6: 1$ to perforate the concrete target plate. Acceleration signal is acquired using the memory and accelerometer in the projectile tail.

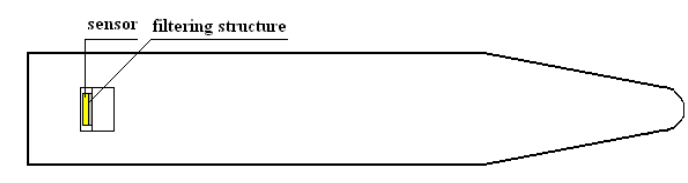

Fig. 2. Sensor and filtering structure of model.

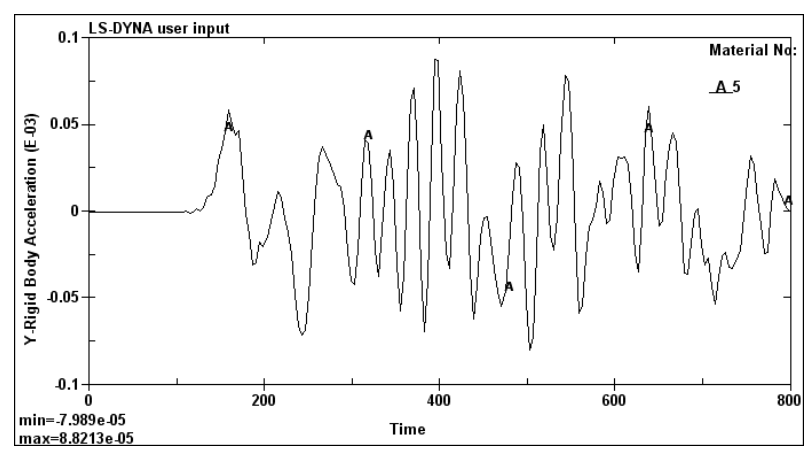

Fig. 3. Original acceleration curve.

Following Fig. 3 the first peak of speed is $59360 \mathrm{~g}$, pulsed width is $50 \mathrm{us}$, maximum peak is $88300 \mathrm{~g}$. There are a lot of noise signal which is produced projectile impelled in the acceleration curve, in order to correctly identify overload information, we must eliminate the high frequency noise that will impact overload signal.

Through the buffer filtering system the acceleration signal of physical filter will be achieved. Changing the parameters of filter material, filtering effect for acceleration signal is studied when the parameters of material change.

There are many factors that affect the acceleration signal, such as muzzle velocity, penetrative angle, position of the sensor, shape of the warhead, ratio of length and diameter, propagation of the stress wave, selection of the filtering material, etc. Many factors have been analyzed before, such as position of the sensor, shape of the warhead, ratio of length and diameter of projectile, etc. In this article, the influence of stress wave's spreading and filtering material to the signal will be studied.

When the projectile strikes the target, a big acceleration is imposed to the nose of projectile suddenly. Because the loading rate is big enough, the propagation of elastic-plastic stress wave will be generated in the projectile, causing the stress, displacement, velocity and acceleration of each mutation to break. Especially for bar type assaulting weapons, the effect of stress wave's spreading on acceleration signal should be taken into account.

When the projectile penetrates the hard objects high-speedily, such as concrete, rock and so on, there are two kinds of stress wave in the projectile: the elastic wave (longitudinal wave, spread along the axis of projectile) and the plastic wave (shear wave, spread along the radial direction of projectile). In the stage of elastic deformation [4], the relationship between the longitudinal wave velocity and the shear wave velocity in a solid is shown in the (3) and (4):

$$
\begin{gathered}
c_{1}=\sqrt{\frac{1-\mu}{(1+\mu)(1-2 \mu)} \frac{E}{\rho}} \\
c_{2}=\sqrt{\frac{1}{2(1+\mu)} \frac{E}{\rho}}
\end{gathered}
$$

In the formulas, $\mathrm{c}_{1}$ and $\mathrm{c}_{2}$ are longitudinal wave velocity and shear wave velocity respectively, and $\rho$ is density, $E$ is elastic modulus, $\mu$ is poisson's ratio.

As shown in the two formulas above, the longitudinal wave velocity and the shear wave velocity are both determined by the material mechanics parameters $\sqrt{\mathrm{E} / \rho}$ and $\mu$, and the ratio of wave velocity is only determined by $\mu$. So deeply investigating the relation between wave velocity and $\mu$ can deepen our understanding of stress wave.

$$
\frac{c_{1}}{c_{2}}=\sqrt{\frac{2(1-\mu)}{1-2 \mu}} \quad(0<\mu<0.5)
$$

Visibly, two kinds of stress wave velocity have the following characteristics. First, the longitudinal wave speed is bigger than the shear wave velocity. Second, the ratio of longitudinal wave speed and shear wave velocity increases gradually with $\mu$ increasing gradually from 0 . When $\mu$ tends to zero, the material is almost rigid completely, namely the 
material is close to the rigid body. When $\mu$ tends to 0.5 , from the definition of poisson's ratio, the ratio of vertical deformation and horizontal deformation is close to 0.5 in the unidirectionally tensile test. This is another limit state in the angle of solid deformation [5].

The waveform of elastic wave changes a little in the process of propagation, so the attenuation of stress can be ignored in the whole process of penetration. However, the waveform of plastic wave changes a lot and attenuates rapidly. The factors which affect the attenuation are quite complicated. The plastic wave velocity is only about $1 / 10$ of the elastic wave velocity in the rod. Due to the stress wave effect, the deformation of projectile is mainly the abrasion of tip. The elastic wave arrives soon at the moment of projectile hitting the target, while the plastic wave arrives soon at the moment of projectile completely entering into the target body. In addition to the stress wave velocity, the stress wave amplitude is also a important characteristic. Only large enough stress wave amplitude can affect the structural object.

\section{OptimizATION}

\section{A. Optimization of the Density Parameters}

By changing the buffer material density, it is studied how the material density influence the acceleration curve. A total of seven group simulation are tried. Remaining other parameters unchanged, density change from $1 \mathrm{~g} / \mathrm{cm}^{3}$ to 1.8 $\mathrm{g} / \mathrm{cm}^{3}$ in simulation. The finishing simulated results are show in Fig. 4.

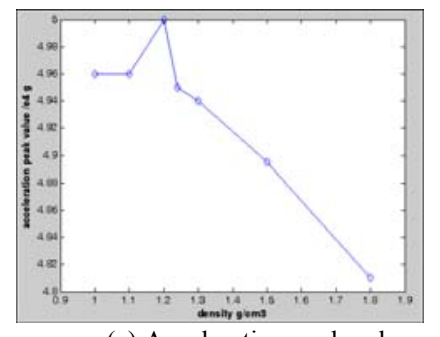

(a) Acceleration peak value

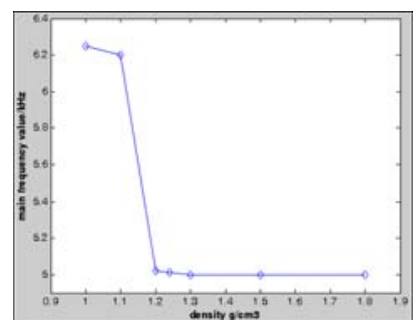

(b) Main frequency value

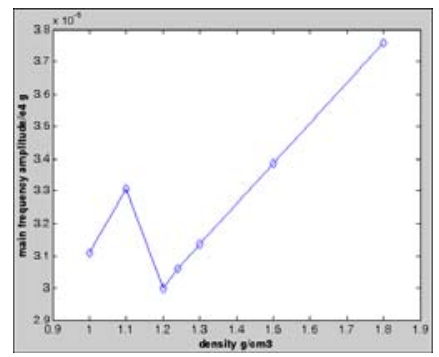

(c) Main frequency amplitude

Fig. 4. Effect of material density for acceleration signal.

Based on the simulation of sensor and buffer system, we analyze the effect of density for acceleration signal. From Fig. 4 (a) with the increase of density, the peak of acceleration buffered change smooth; the main frequency value have a sharp decline in the density among $1.1 \mathrm{~g} / \mathrm{cm}^{3}$ to $1.2 \mathrm{~g} / \mathrm{cm}^{3}$; the main frequency amplitude rise after drop.

After analyzing and screening from many simulation results, we select the material of density of $1.2 \mathrm{~g} / \mathrm{cm}^{3}$ and Young's modulus of $120 \mathrm{MPa}$ to simulate. The acceleration curve is following.

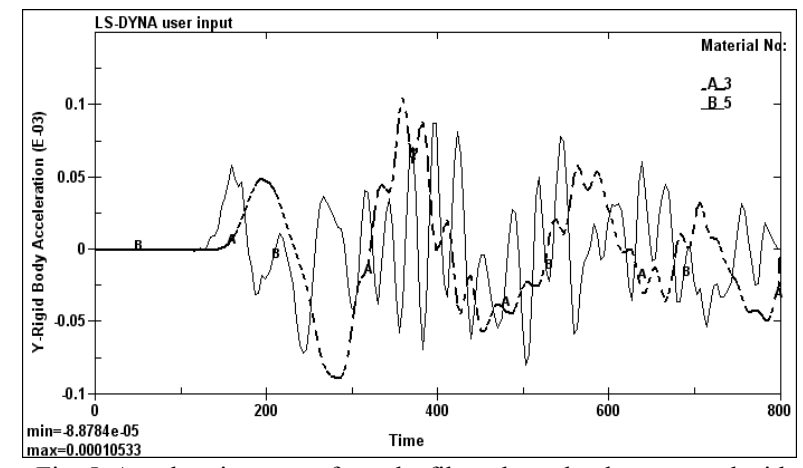

Fig. 5. Acceleration curve from the filtered overloads compared with the corresponding original values.

In Fig. 5 we can see that the curve change smooth after using buffer. Original rigid body overload pulse is $50 u$ s, and filtered pulse is $86 u$ s. The oscillation signal disappears on curve.

\section{B. Optimization of the Young's Modulus Parameters}

Other parameters constant, by changing Young's modulus of the filter material, we focus our attention on the effects of Young's modulus for acceleration signal, which a short projectile experiences during its penetration into a target.

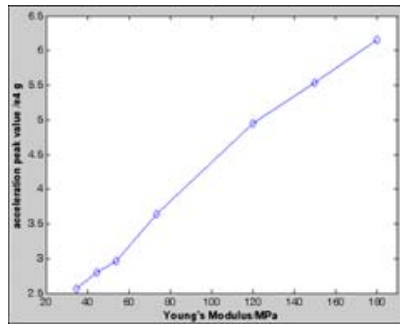

(a) Acceleration peak value

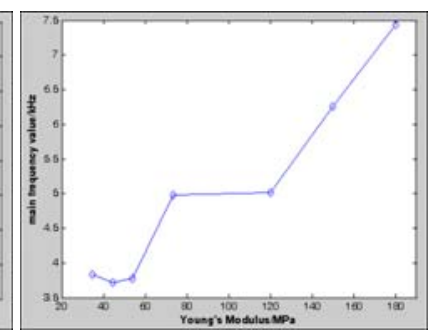

(b) Main frequency value

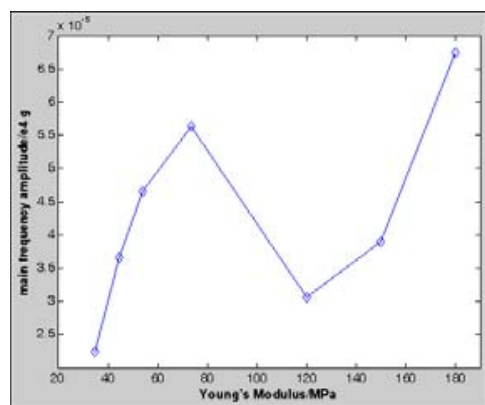

(c) Main frequency amplitude

Fig. 6. Effect of material Young's modulus for acceleration signal.

In Fig. 6 we can see that acceleration rigid overload peak reduces with Young's modulus of filtering material to decrease. Both Young's modulus and acceleration peak have a linear relationship in the test range. With the decrease of the Young's modulus, acceleration main frequency value is also reduced, and the main frequency amplitude increase after reduce. Visibly, if Young's modulus of filtering material is so small or large that rigid body overload peak will change. Finding suitable materials had a befitting Young's modulus as filtering materials is to ensure that the rigid body signal is perfectly recognized, and high frequency noise signal is filtered [6].

When the elastic modulus is $34.5 \mathrm{MPa}$, the curve about the base and the acceleration of acceleration sensor are drawn as shown in Fig. 7. 


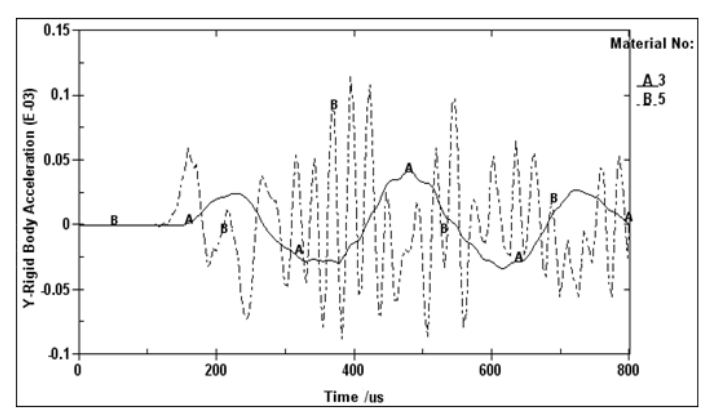

Fig. 7. Time course curve on base and acceleration of sensor steel shell when the elastic modulus is $34.5 \mathrm{MPa}$.

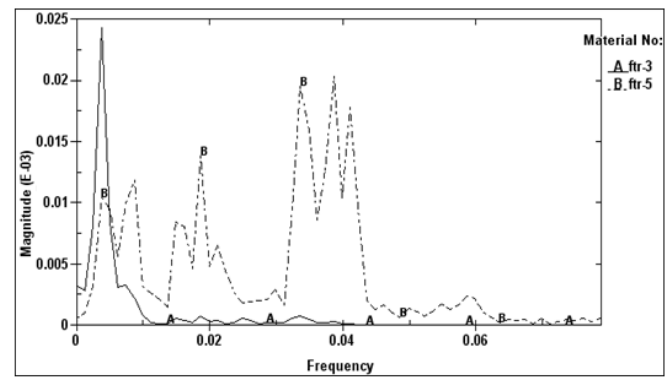

Fig. 8. Spectrum diagram on base and acceleration of sensor steel shell when elastic modulus is $34.5 \mathrm{Mpa}$.

As shown in Fig. 8, it is the first wave crest of dotted line when the first peak arrives outside base. The pulse width of wave becomes wider go through the buffering material. When the wave spreads to the sensor steel shell, amplitude has fallen by half, from $58000 \mathrm{~g}$ to $25700 \mathrm{~g}$. Then the peak value is in the bearable range of the acceleration sensor. As shown in the acceleration spectrum diagram, frequency amplitude is significantly weakened after $20 \mathrm{KHZ}$.

As mentioned before, the bigger the ratio of damping layer and substrate thickness is, the greater the structural lossy factor is. Therefore, if we want to study the influence of the thickness of filtering material to the acceleration signal, we just need investigate the effect of the thickness on the filtering result. In this paper, we study the influence of the filter with the thickness of $0.2 \mathrm{~cm}, 0.3 \mathrm{~cm}$ and $0.4 \mathrm{~cm}$ to the acceleration curve of acceleration sensor.

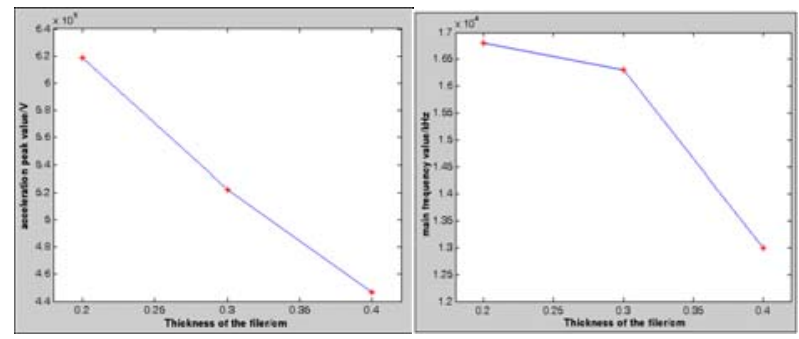

(a) Acceleration peak value

(b) Main frequency value

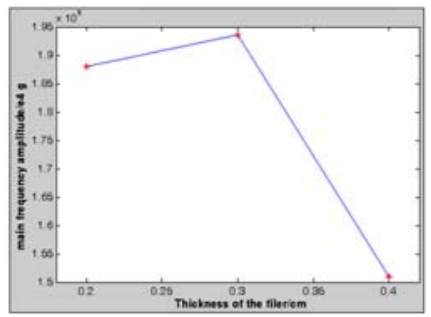

(c) Main frequency amplitude

Fig. 9. The effect of the thickness of gasket material on the acceleration signal.
As shown in Fig. 9, the first peak value of the acceleration curve is decreasing with the increase of the thickness of the filter. This is true in theory. The impact of buffering material on the whole system increases with the increase of the thickness of the filter, so that the main frequency of the curve on frequency and amplitude decreases. With the increase of thickness, the main frequency amplitude increases first and decreases later. Then the distance between the natural frequency of the filter and the sensor also has a trend of increasing first and decreasing later, so the phenomenon of the amplitude increasing first and decreasing later is shown. This is caused by the resonance of filter and sensor. So it is good for the filtering effect of sensor to select the thickness around $0.3 \mathrm{~cm}$.

The filter with the thickness of $0.3 \mathrm{~cm}$ is carried on the numerical analysis and the results are shown in Fig.10.

As shown in Fig. 10, after adding the filter, the first wave peak of the sensor is almost no change, but the pulse width is widened. This is advantageous to the signal identification of sensor. Due to adding the filter, the acceleration curve is smooth and the problem of peak surging caused by the high frequency oscillation is eliminated. As shown in Fig.11, after adding the filter, frequency amplitude is significantly weakened after $20 \mathrm{KHZ}$ and it is weakened by $1 / 10$ of the original. It has reached the desired filtering effect.

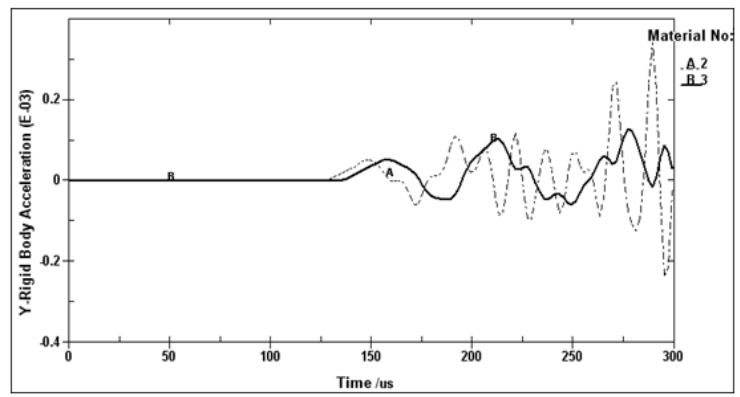

Fig. 10. Time course curve on base and acceleration of sensor steel shell when the thickness of filter is $0.3 \mathrm{~cm}$.

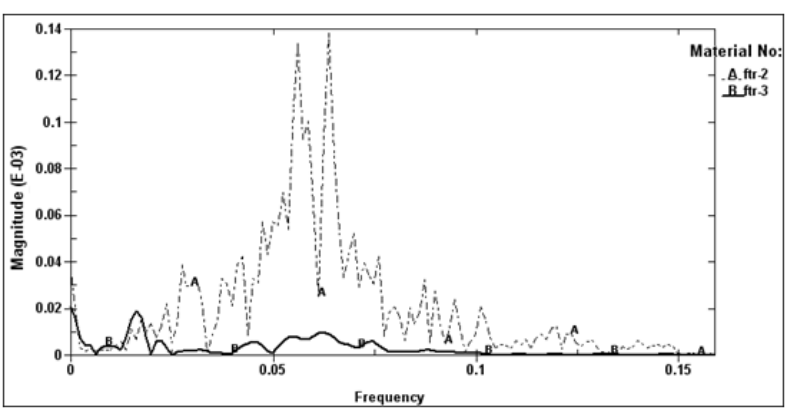

Fig. 11. Spectrum diagram of base and acceleration on sensor steel shell when the thickness of filter is $0.3 \mathrm{~cm}$.

\section{CONCLUSION}

Through established the mode of the penetrating concrete target plate of projectile, we gives out the filter mat had different material parameters.

The results show that there is a great influence for sensor acceleration signal by material density and Young's modulus of the filtering mat. With the increase of the density, the peak acceleration drop using buffer, main frequency value 
increase, amplitude decrease. With the increase of the Young's modulus, the peak acceleration are gradually increasing using buffer, the main frequency value changes according curve, amplitude is more nonlinear.

The material of density of $1.2 \mathrm{~g} / \mathrm{cm}^{3}$ and Young's modulus of $120 \mathrm{MPa}$ is selected to use as a filter for sensor in high-overload environment.

\section{REFERENCES}

[1] B. Zhang and G. C. Shi, "Mechanical filtering for target recognition of hard target penetration," Journal of Detection and Control, vol. 32, 2010.

[2] J. Peng, "High-G impact test value of the research of key technologies," M. S. thesis, North University of China, 2009

[3] Y. B. Shi, Z. Q. Zhu, X. P. Liu, K. Du, and J. Liu, "Design and impact analysis of a high-g aecelerometer," Explosion and shock waves, vol. 30, pp. 329-332, 2010.
[4] H. P. Liu, S. Q. Gao, and L. Jin, "Experiment and simulation for projectile penetrating concrete target with finite thickness," Acta Armamentarll.

[5] G. M. Xie, Oscillate mechanics, Publishing Company of National Defense Industry, 2007.

[6] H. B. Li, "Oscillate theory and engineering application," Publishing Company of Beijing Institute of Technology, 2006.

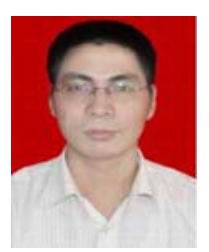

Yunbo Shi received Master of Engineering in Electronic Science and Technology, North University of China, Taiyuan, Shan'xi, April 2003. He was Associate professor of North University of China. He mainly engaged in the research of MEMS and the micro inertial device etc. 